\title{
Modelling and Analysis of Wi-Fi and LAA Coexistence with Priority Classes
}

\author{
Anand M. Baswade*, Luca Beltramelli ${ }^{\dagger}$, Antony Franklin A*, Mikael Gidlund ${ }^{\dagger}$, Bheemarjuna Reddy Tamma*, \\ and Lakshmikanth Guntupalli ${ }^{\dagger}$ \\ *Department of Computer Science and Engineering Indian Institute of Technology Hyderabad, India \\ \{cs14resch11002, antony.franklin, tbr\}@iith.ac.in \\ ${ }^{\dagger}$ Department of Information Systems and Technology Mid Sweden University, Sweden \\ \{luca.beltramelli, mikael.gidlund, lakshmikanth.guntupalli\}@miun.se
}

\begin{abstract}
The Licensed Assisted Access (LAA) is shown as a required technology to avoid overcrowding of the licensed bands by the increasing cellular traffic. Proposed by 3GPP, LAA uses a Listen Before Talk (LBT) and backoff mechanism similar to Wi-Fi. While many mathematical models have been proposed to study the problem of the coexistence of LAA and Wi-Fi systems, few have tackled the problem of QoS provisioning, and in particular analysed the behaviour of the various classes of priority available in $\mathrm{Wi}-\mathrm{Fi}$ and LAA. This paper presents a new mathematical model to investigate the performance of different priority classes in coexisting Wi-Fi and LAA networks. Using Discrete Time Markov Chains, we model the saturation throughput of all eight priority classes used by Wi-Fi and LAA. The numerical results show that with the 3GPP proposed parameters, a fair coexistence between Wi-Fi and LAA cannot be achieved. Wi-Fi users in particular suffer a significant degradation of their performance caused by the collision with LAA transmissions which has a longer duration compared to Wi-Fi transmissions.
\end{abstract}

\section{INTRODUCTION}

LTE in unlicensed spectrum (LTE-U) is a promising solution proposed in the literature to improve cellular data rates and reduce traffic load on the licensed spectrum. The deployment of small cell LTE-U base station (eNB) and Wi-Fi Access Point (AP) in unlicensed spectrum creates new challenges like fair coexistence along with efficient utilization of unlicensed spectrum. Two possible solutions have been proposed to allow a fair coexistence of LTE with Wi-Fi. One solution is to use ON-OFF cycles [1] (i.e., duty cycle) in LTE and the other solution is to adopt a Listen Before Talk (LBT) [2] mechanism in LTE similar to Wi-Fi. The LTE with LBT mechanism is globally accepted and standardized by 3GPP under the name Licensed Assisted Access (LAA) [2]. In literature [1], [3] and [4] have shown that LAA can be fair with the Wi-Fi Distributed Coordination Function (DCF) mechanism. But, the effect on Quality of Service (QoS) traffic in Wi-Fi and LAA coexistence scenario is still a major concern that has not yet received much attention.

With IEEE 802.11e [5], to handle the multimedia traffic, such as voice and real-time video, the Wi-Fi research community introduced support for QoS differentiation. In IEEE 802.11e, the Enhanced Distributed Channel Access (EDCA) was introduced; an extension of the DCF protocol of Wi-Fi with QoS support. In EDCA, Wi-Fi traffic is assigned to one of the four Access Categories (ACs). To prioritize some type of traffic over others, EDCA uses different values of Arbitrary Inter Frame Space (AIFS), Contention Window (CW), and Transmission Opportunity (TXOP) for each AC. The values of the EDCA parameters can be found in Table I. Similarly, LAA also provides QoS by defining four priority classes with parameters given in Table II. By comparing the parameters of Table I and Table II, it can be noted that there are few differences between LAA and Wi-Fi.

In this paper, we focus on the performance evaluation of EDCA and LAA priority classes. In the literature, performance evaluation of Wi-Fi and LAA has been carried out either using simulations [6], [7], [8] or analytical models [9], [10], [11], but never considering different priority classes.

To the best of the authors' knowledge no one has considered the case in which multiple priority classes are concurrently used in both Wi-Fi and LAA networks. To address this, we propose a new mathematical model for LAA and Wi-Fi coexistence capable of describing the performance of all eight priority classes of EDCA and LAA. The main contributions of this paper can be summarized as follows:

1) We provide an analytical framework to evaluate the throughput of coexisting LAA and Wi-Fi networks with traffic of different priority classes. The model captures the effects of transmission durations and message structure of LAA and Wi-Fi systems on the network throughput.

2) We show that LAA is unfair with Wi-Fi in terms of throughput. Further, we find that the causes of this unfairness are the different transmission durations of LAA and Wi-Fi priority classes.

3) We show that Wi-Fi throughput decreases when RTS/CTS is enabled for Wi-Fi, while LAA classes increase their throughput.

4) Finally, to improve the fairness between Wi-Fi and LAA systems, we propose to reduce the LAA transmission opportunity.

The remainder of the paper is organized as follows: Section II presents the related work and the existing mathematical models for coexistence between Wi-Fi an LAA. In Section III, the proposed mathematical model is presented. The results 
obtained from the mathematical model are reported in Section IV. Conclusions are provided in Section V.

\section{RELATED WORK}

In recent years there has been a lot of effort to study how to make LTE operate in the unlicensed spectrum without being unfair towards other technologies in unlicensed bands, namely Wi-Fi. In [3], it has been shown that directly using LTE in the unlicensed spectrum significantly degrades the performance of neighbouring Wi-Fi users. The schedule based transmission of LTE needs to be changed if we want to have fairness in the unlicensed band. LBT [2], [12] and CSAT [1] methods have been proposed to allow LTE to fairly coexist with Wi-Fi. For a in-depth analysis of the benefits and challenges of LTE and Wi-Fi coexistence in unlicensed band, we refer the reader to [13]. In [9] and [11] mathematical models of Wi-Fi and LAA have been proposed based on the famous Bianchi model [14]. In all of them the authors studied the problem of coexisting Wi-Fi and LAA nodes without considering different priority classes and different transmission durations. Moreover, previous works have underestimated LAA throughput by assuming that in case of a collision with Wi-Fi the whole frame would be lost. In reality, we argue that in case of collision with short Wi-Fi transmissions only the first subframe would be lost. In [10], an analytical framework was proposed for analysing the throughput and the MAC delay distribution of coexisting Wi-Fi-LAA users. The authors consider a mechanism for dynamically adapting the contention window size of LAA which is different from the binary exponential backoff of Wi-Fi. Once again, the model does not account for the presence of different priority classes in Wi-Fi or LAA. In [15], a mathematical model is proposed for the coexistence analysis of Wi-Fi DCF and LAA with priority classes but without considering Wi-Fi EDCA.

TABLE I: Wi-Fi EDCA Parameters [5]

\begin{tabular}{c|c|c|c|c|c|c}
\cline { 2 - 7 } & Class & \multicolumn{2}{c}{$\mathrm{CW}^{\mathrm{MIN}}$} & \multicolumn{2}{c}{ CW $^{\mathrm{MAX}}$} & \multicolumn{2}{c}{ AIFSN } & \multicolumn{2}{c}{$\begin{array}{c}\text { Retry Limit } \\
(\mathrm{M})\end{array}$} & \multirow{2}{*}{ TXOP } \\
\hline $\mathrm{AC}[3]$ & $\mathrm{BK}$ & 15 & 1023 & 7 & $7 / 4$ & 1 frame \\
\hline $\mathrm{AC}[2]$ & BE & 15 & 1023 & 3 & $7 / 4$ & 1 frame \\
\hline $\mathrm{AC}[1]$ & VI & 7 & 31 & 2 & $7 / 4$ & $3.008 \mathrm{~ms}$ \\
\hline $\mathrm{AC}[0]$ & VO & 3 & 15 & 2 & $7 / 4$ & $1.504 \mathrm{~ms}$ \\
\hline
\end{tabular}

TABLE II: LAA Downlink Channel Access Parameters [2]

\begin{tabular}{|c|c|c|c|c|c|}
\hline $\begin{array}{l}\text { Priority } \\
\text { Class }\end{array}$ & $\mathrm{CW}^{\mathrm{MIN}}$ & $\mathrm{CW}^{\mathrm{MAX}}$ & $m$ & $\begin{array}{l}\text { Retry Limit } \\
\text { at } \mathrm{CW}^{\mathrm{MAX}}\end{array}$ & $\begin{array}{c}\text { Transmission Duration } \\
\Gamma\end{array}$ \\
\hline 4 & 15 & 1023 & 7 & 4 & $8 \mathrm{~ms}$ \\
\hline 3 & 15 & 63 & 3 & 4 & $8 \mathrm{~ms}$ \\
\hline 2 & 7 & 31 & 1 & 4 & $3 \mathrm{~ms}$ \\
\hline 1 & 3 & 15 & 1 & 4 & $2 \mathrm{~ms}$ \\
\hline
\end{tabular}

\section{WI-FI EDCA AND LAA AC COEXISTENCE}

ANALYTICAL MODEL

For the sake of clarity, the proposed analytical model presented below has been conveniently divided in multiple sections. The Discrete Time Markov Chain used to model the performance of Wi-Fi EDCA protocol and LAA is first explained in Section III-A. In Section III-B, we present the steps to calculate the transmission probabilities. In Section III-C, the probabilities of successful and failed transmission attempts are derived for both Wi-Fi and LAA. Section III-D provides the formulas for calculating the throughput for each of the priority classes in Wi-Fi and LAA. Section III-E presents the formulation used to calculate the fairness index metric between Wi-Fi and LAA. Section III-F closes the mathematical model by studying the case in which Wi-Fi uses the optional RTS/CTS mechanism. In the context of this paper, we make use of the following assumptions:

- Only downlink traffic from the eNB to the LAA users is present in the network;

- The channel is assumed to be ideal, i.e., packet loss is only caused by collisions;

- No frame capture effect at the receivers;

- All nodes (LAA eNB and Wi-Fi AP) are within the carrier sense range, i.e., there are no hidden nodes;

- Saturated traffic condition at all nodes;

- LAA and EDCA use the same slot time $\sigma$.

TABLE III: Notation used in the analytical model

\begin{tabular}{|l|l|}
\hline Notation & Description \\
\hline \hline$C W_{i, r}$ & Contention window size \\
$\sigma$ & Slot duration \\
$M_{i}$ & Maximum retransmission attempts \\
$P C_{i}$ & Collision probability \\
$P B_{i}$ & Backoff countdown blocking probability \\
$b_{i, r, z}$ & Steady state probability of $(i, r, z)$ \\
$\tau_{i}$ & Transmission Probability \\
\hline \hline & EDCA \\
\hline AIFSN $i$ & Adaptive inter frame space number \\
SIFS & Short inter frame space \\
$n_{w}$ & Number of Wi-Fi devices \\
$N_{i}$ & Number of Wi-Fi packets in a TXOP \\
$T_{p h y}$ & Phy overhead transmission time \\
$D_{m a c}$ & Size of MAC overhead \\
$D_{a c k}$ & Size of ACK frame \\
$D_{d a t a}$ & Size of data payload \\
$B R$ & Base transmission rate \\
$D R$ & Data transmission rate \\
\hline \hline & LAA \\
\hline$n_{l}$ & Number of LAA devices \\
$\Gamma_{i}$ & Duration of an LAA Class $i$ transmission \\
$T_{l}$ & Half of a LTE slot duration \\
$T_{s f r a m e}$ & Duration of a LTE sub-frame \\
\hline
\end{tabular}

\section{A. Discrete Time Markov Chain Analysis}

Wi-Fi nodes and LAA nodes access the channel using similar CSMA/CA mechanisms. Wi-Fi uses the DCF/EDCA mechanism, consisting of a random access with binary exponential backoff. In LAA, the channel access procedure proposed by $3 \mathrm{GPP}$ is also based on random access with binary exponential backoff. Each technology defines four priority classes of channel access. According to IEEE 802.11 and LAA, when a node has data to transmit, it will randomly choose for its transmission attempt a backoff slot between zero and the current contention window size. After each transmission, the size of the contention window is updated according to a truncated binary exponential backoff. The size of the contention window for a packet with priority Class $i$, 


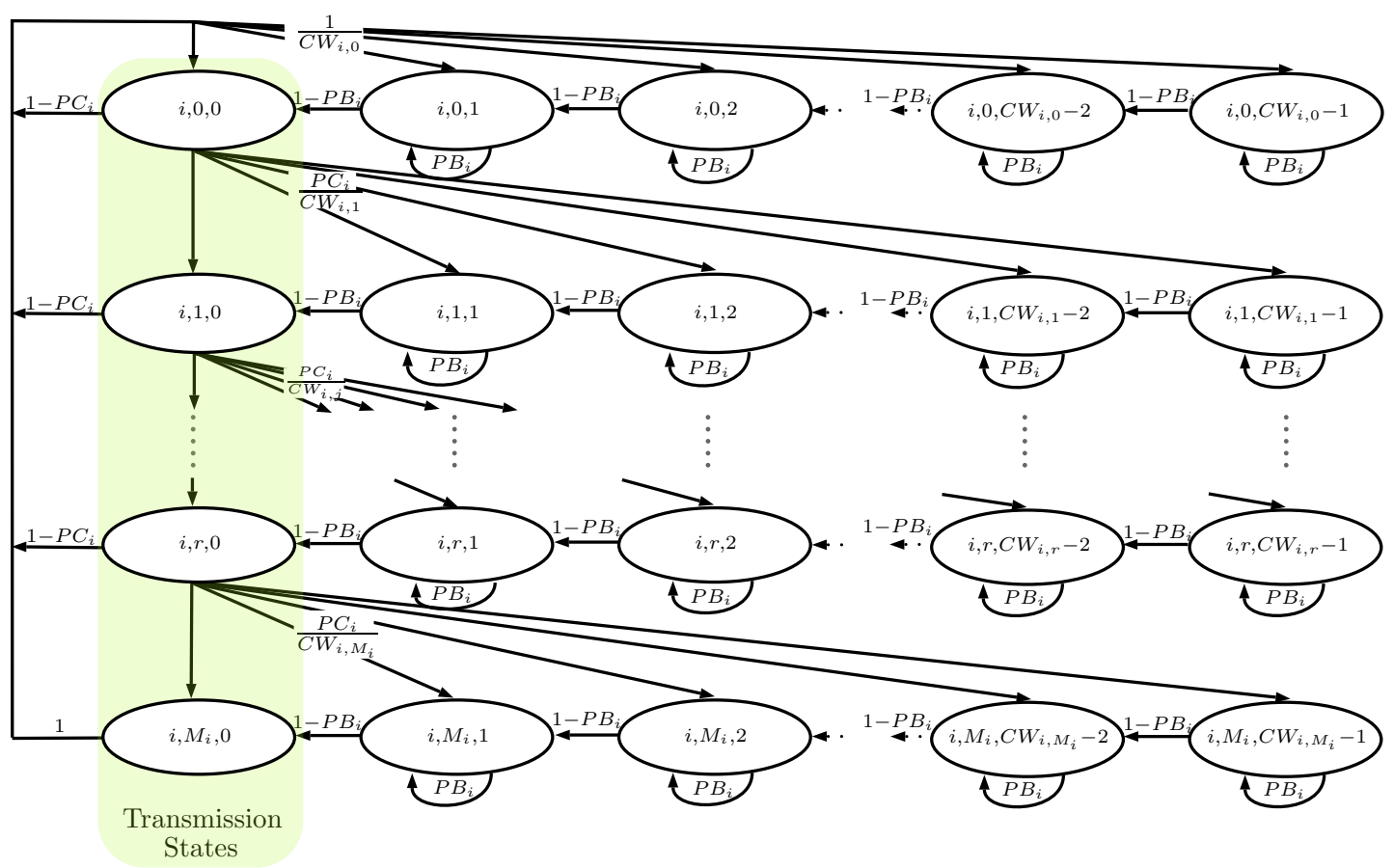

Fig. 1: DTMC model for Wi-Fi EDCA and LAA LBT.

after experiencing $r$ unsuccessfully transmission attempts is

$$
\mathrm{CW}_{i, r}=\min \left\{2^{r} \cdot \mathrm{CW}_{i}^{\mathrm{MIN}}, \mathrm{CW}_{i}^{\mathrm{MAX}}\right\} \text { for } r=0, \ldots, M_{i},
$$

where $M_{i}, \mathrm{CW}_{i}^{\mathrm{MAX}}$, and $\mathrm{CW}_{i}^{\mathrm{MIN}}$ are respectively the maximum number of retransmissions, the maximum and minimum contention window size for priority Class $i$. After sensing the medium as busy, both Wi-Fi and LAA prescribes that each priority class senses an idle medium for different time before decrementing its backoff counter. In EDCA, this sensing time is called the Arbitration Inter Frame Space (AIFS), given by $\operatorname{AIFS}_{i}=\operatorname{SIFS}+\operatorname{AIFSN}_{i} \cdot \sigma$, where, SIFS is the Short Inter Frame Space, $\sigma$ is the slot time and AIFSN is the AIFS number, given in Table I. In LAA the same concept is called Defer Duration $T_{d}$, given by $T_{d}=T_{f}+\left(m_{i}+1\right) \cdot \sigma$, where, $m_{i}$ and $T_{f}$, given in Table II, are respectively the equivalent in LAA of the AIFSN and SIFS of Wi-Fi. According to 3GPP, the slot time $\sigma$ is common to both Wi-Fi and LAA and the duration of an SIFS and $T_{f}$ are equal. Once a node has gained access to the channel the amount of time available for the communication depends on the priority of the class. In IEEE 802.11, this time is called a transmission opportunity (TXOP). Similarly, in LAA we have the maximum transmission time of the carrier signal $\left(\Gamma_{i}\right)$. The values of these parameters are shown in Table I and II.

To study the performances of Wi-Fi EDCA mechanism and the Listen Before Talk (LBT) mechanism of LAA, we use a 3-dimensional Discrete Time Markov Chain (DTMC) similar to the one used in [16], from which the unsaturated states were removed. The DTMC showed in Fig. 1 can capture the behaviour of both Wi-Fi and LAA nodes in saturation condition.
A generic state of the DTMC is identified by the 3-tuple $(i, r, z)$, index $i$, identifies the priority class of the message; $r$, the number of retransmission attempts; $z$ the value of the backoff counter. The transition probabilities between the states of the DTMC in Fig. 1 are given by

$$
\begin{cases}P(i, 0, z \mid i, r, 0)=\frac{\left(1-P C_{i}\right)}{\mathrm{CW}_{i, 0}} & \text { for } 0 \leq z \leq \mathrm{CW}_{i, 0}-1 \\ P\left(i, 0, z \mid i, M_{i}, 0\right)=\frac{1}{\mathrm{CW}_{i, 0}} & \text { for } 0 \leq z \leq \mathrm{CW}_{i, 0}-1 \\ P(i, r, z-1 \mid i, r, z)=1-P B_{i} & \text { for } 1 \leq z \leq \mathrm{CW}_{i, r}-1 \\ P(i, r+1, z \mid i, r, 0)=\frac{P C_{i}}{\mathrm{CW}_{i, r+1}} & \text { for } 0 \leq z \leq \mathrm{CW}_{i, r+1}-1\end{cases}
$$

for $0 \leq r \leq M_{i}-1$. Where $P C_{i}$ and $P B_{i}$ are respectively the collision probability and the backoff freezing probability for priority Class $i$. Let $b_{i, r, z}$ be the stationary probability for the state $(i, r, z)$. From the analysis of the DTMC, we obtain the following system of equations

$$
\left\{\begin{array}{l}
b_{i, 0,0}=b_{i, M_{i}, 0}+\left(1-P C_{i}\right) \sum_{r=0}^{M_{i}-1} b_{i, r, 0} \\
b_{i, r, 0}=\left(P C_{i}\right)^{r} b_{i, 0,0} \\
b_{i, r, z}=\frac{C W_{i, r}-z}{C W_{i, r}} \frac{b_{i, r, 0}}{1-P B_{i}}
\end{array}\right.
$$

The normalization property of the DTMC gives:

$$
\sum_{r=0}^{M_{i}} b_{i, r, 0}+\sum_{r=0}^{M_{i}} \sum_{z=1}^{C W_{i, r}-1} b_{i, r, z}=1 .
$$

By solving the system of equations of (2) and (3), we can obtain the expression of the transmission probability of a Class $i$ as

$$
\tau_{i}=\sum_{r=0}^{M_{i}} b_{i, r, 0}
$$




\section{B. Wi-Fi and LAA Transmission Probabilities}

The transmission probabilities of Wi-Fi $\left(\tau_{i}^{w}\right)$ and LAA $\left(\tau_{i}^{l}\right)$ calculated using (4) are expressed in term of $P C_{i}$ and $P B_{i}$. To find the probabilities of various events on the shared channel, we exploit the independence of Wi-Fi and LAA transmission probabilities. From here on, we assume that higher priority classes have a smaller index $i$, that is, when writing $j \geq i$ with $j, i=0, \ldots, 3$, we include any class of lower or equal priority than $i$. For a given Wi-Fi or LAA node, transmitting data of Class $i$, the collision probability in a time slot is the probability that at least one other node transmits in the same timeslot (External Collision) or the same node has a higher priority data, scheduled for transmission in the same timeslot (Internal Collision). The collision probability of a Wi-Fi node, transmitting traffic of Class $i$, is given by

$P C_{i}^{w}=1-\left(1-\sum_{k=0}^{3} \tau_{k}^{l}\right)^{n_{l}} \prod_{j \geq i}\left(1-\tau_{j}^{w}\right)^{n_{w}-1} \prod_{s<i}\left(1-\tau_{s}^{w}\right)^{n_{w}}$

The collision probability of an LAA node, transmitting traffic of Class $i$, is given by

$$
\begin{aligned}
P C_{i}^{l}= & 1-\left[\left(1-P_{\mathrm{fc}}\right)+P_{\mathrm{fc}}\left(1-\sum_{k=0}^{3} \tau_{k}^{w}\right)^{n_{w}}\right] \times \\
& \prod_{j \geq i}\left(1-\tau_{j}^{l}\right)^{n_{l}-1} \prod_{s<i}\left(1-\tau_{s}^{l}\right)^{n_{l}} .
\end{aligned}
$$

Where $n_{w}$ and $n_{l}$ are respectively the number of Wi-Fi and LAA nodes. In LAA, data transmissions are always aligned to $0.5 \mathrm{~ms}$ of LAA time slot boundaries [4]. After following LBT, when an LAA node gain access to the channel, it transmits a reservation signal to reserve the channel (i.e., with the sole purpose of preventing other nodes from transmitting) till the slot boundary after which LAA can transmit the actual signal. In such case, sometimes a Wi-Fi packet may collide with the LAA reservation signal. Thus in (6), $P_{\mathrm{fc}}$ is the probability that a collision between Wi-Fi and LAA occurs before the start of an LAA time slot (i.e, before actual LAA data signal transmission). $P_{\mathrm{fc}}$ is equal to the ratio between a Wi-Fi packet transmission time and LAA slot time (i.e., $0.5 \mathrm{~ms}$ ).

For a given Wi-Fi or LAA node transmitting data of Class $i$, the backoff countdown blocking probability is the probability that the node will not decrease its backoff counter because the medium is sensed busy. This probability depends on the duration of the Clear Channel Assessment (CCA) time defined by Wi-Fi and LAA standards. Let $x \in\{w, l\}$ the index indicating the Radio Access Technology (RAT) of the node, i.e., Wi-Fi $(w)$ or an LAA $(l)$. With $\bar{x}$, we indicate the complement of $x$ (i.e. if $x=w, \bar{x}=l$ and vice versa). Let $\mathrm{CCA}_{i}^{x}$ be the number of slots that a node with RAT $x$ transmitting Class $i$ traffic, has to sense the channel before start decrementing the backoff counter.

$$
\mathrm{CCA}_{i}^{x}= \begin{cases}\operatorname{AIFSN}_{i} & \text { for } x=w \\ m_{i}+1 & \text { for } x=l\end{cases}
$$

Let $\mathrm{CCA}_{\min }$ be the minimum number of slots of CCA among Wi-Fi and LAA priority classes, $\mathrm{CCA}_{\min }=\min \left\{\mathrm{CCA}_{i}^{x}\right\}$. According to [16], the backoff countdown blocking probability $P B_{i}^{x}$ is given by

$$
\begin{aligned}
P B_{i}^{x}= & 1-\left[\left(1-\sum_{k=0}^{3} \tau_{k}^{\bar{x}}\right)^{n_{\bar{x}}}\left(1-\tau_{i}^{x}\right)^{n_{x}-1} \times\right. \\
& \left.\prod_{j=0, j \neq i}^{3}\left(1-\tau_{j}^{x}\right)^{n_{x}}\right]^{\mathrm{CCA}_{i}^{x}-\mathrm{CCA}_{\min }+1} .
\end{aligned}
$$

where $n_{x}$ and $\tau^{x}$ are respectively the total number of nodes and the transmission probability of RAT $x$. Combining (4), (5), (6), and (8), we obtain a non-linear system of equations that can be solved using numerical methods for $\tau_{i}^{w}, \tau_{i}^{l}, P C_{i}^{w}$, $P C_{i}^{l}, P B_{i}^{w}$, and $P B_{i}^{l}$.

\section{Average Duration of a Contention Slot}

To calculate the throughput of Wi-Fi and LAA in a coexistence scenario, we are first required to calculate the success and collision probabilities in a contention slot. Depending on the number of nodes transmitting, a contention slot can contain one of the following events: a) an idle slot; b) a successful transmission; c) a collision. Successful transmission and collision events can be subdivided according to the class and RAT that causes them.

Idle Slot: The probability that the medium is idle, is the probability that no Wi-Fi or LAA node transmits

$$
P_{\mathrm{idle}}=\left(1-\sum_{i=0}^{3} \tau_{i}^{w}\right)^{n_{w}}\left(1-\sum_{i=0}^{3} \tau_{i}^{l}\right)^{n_{l}} .
$$

Successful Transmission: The probability of successful transmission in a contention slot is the probability that only one node accesses the channel. For a node with RAT $x$ and Class $i$, the probability of successful transmission is given by:

$P S_{i}^{x}=n_{x} \tau_{i}^{x}\left(1-\sum_{k=0}^{3} \tau_{k}^{\bar{x}}\right)^{n_{\bar{x}}} \prod_{j \geq i}\left(1-\tau_{j}^{x}\right)^{n_{x}-1} \prod_{s<i}\left(1-\tau_{s}^{x}\right)^{n_{x}}$.

Collision: A contention slot contains a collision if there are more than one node transmitting. According to the RAT and priority class of the transmitting nodes causing the collision, different amount of channel time is wasted. In a collision involving only Wi-Fi transmissions, the wasted channel time is equal to the transmission time of the first frame in a TXOP. Whereas in LAA, the wasted channel time depends on the maximum transmission time of the carrier signal of the classes involved in the collision. We identify four types of collisions each with different time duration: the collision between Wi-Fi transmissions $\left(P C^{w w}\right)$, the collision between LAA and Wi-Fi $\left(P C^{w l}\right)$, the collision between LAA transmissions of the same priority class $\left(P C^{l_{i} l_{i}}\right)$, and the collision between LAA transmission of different priority classes $\left(P C^{l_{i} l_{j}}\right)$. 
The probability of a collision involving only Wi-Fi nodes is given by

$$
\begin{aligned}
P C^{w w}= & \left(1-\sum_{i=0}^{3} \tau_{i}^{l}\right)^{n_{l}}\left[1-\left(1-\sum_{i=0}^{3} \tau_{i}^{w}\right)^{n_{w}}\right. \\
& \left.-n_{w} \sum_{i=0}^{3} \tau_{i}^{w}\left(1-\sum_{i=0}^{3} \tau_{i}^{w}\right)^{n_{w}-1}\right],
\end{aligned}
$$

where the first term is the probability that there are no LAA transmissions and the second term is the probability that there are at least two Wi-Fi transmissions. The probability of a collision involving one or more $\mathrm{Wi}-\mathrm{Fi}$ transmissions and one LAA transmission of Class $i$ is given by

$$
\begin{aligned}
P C^{w l_{i}}= & {\left[1-\left(1-\sum_{k=0}^{3} \tau_{k}^{w}\right)^{n_{w}}\right] \times } \\
& n_{l} \tau_{i}^{l} \prod_{j \geq i}\left(1-\tau_{j}^{l}\right)^{n_{l}-1} \prod_{s<i}\left(1-\tau_{s}^{l}\right)^{n_{l}} .
\end{aligned}
$$

where the first term is the probability of at least one Wi-Fi transmission and the second term is the probability of having one LAA Class $i$ transmission. The collision probability between two or more LAA Class $i$ transmissions is given by

$$
\begin{aligned}
P C^{l_{i} l_{i}}= & \sum_{k=2}^{n_{l}}\left(\begin{array}{c}
n_{l} \\
k
\end{array}\right)\left(\tau_{i}^{l}\right)^{k} \prod_{j<i}\left(1-\tau_{j}^{l}\right)^{k} \times \\
& {\left[\sum_{s<i}\left(\tau_{s}^{l} \prod_{p<s}\left(1-\tau_{p}^{l}\right)\right)+\prod_{u=0}^{3}\left(1-\tau_{u}^{l}\right)\right]^{n_{l}-k} }
\end{aligned}
$$

$P C^{l_{i} l_{i}}$ is calculated as the probability of having two or more transmissions from LAA Class $i$ and no transmissions from LAA with priority class less than $i$. The probability of a collision involving two or more LAA transmissions of Class $i$ and one LAA transmission of Class $j$ (with $i<j$ ) is given by

$$
\begin{aligned}
P C^{l_{i} l_{j}}= & \sum_{k=1}^{n_{l}-1}\left(\begin{array}{c}
n_{l} \\
k
\end{array}\right)\left(\tau_{i}^{l}\right)^{k} \prod_{s<i}\left(1-\tau_{s}^{l}\right)^{k}\left(n_{l}-k\right) \tau_{j}^{l} \prod_{u<j}\left(1-\tau_{u}^{l}\right) \\
& \times\left(\sum_{t<i}\left[\tau_{t}^{l} \prod_{p<t-1}\left(1-\tau_{p}^{l}\right)\right]+\prod_{v=0}^{3}\left(1-\tau_{v}^{l}\right)\right)^{n_{l}-k-1}
\end{aligned}
$$

The average duration of a contention slot $T_{\mathrm{cs}}$ is calculated by multiplying the probability of each event (9)-(14) by their channel occupancy as given below.

$$
\begin{aligned}
T_{\mathrm{cs}}= & \sum_{i=0}^{3} P S_{i}^{w}\left(\min \left[\mathrm{AIFS}_{i}\right]+T_{s}^{w} \cdot N_{i}-S I F S\right) \\
& +\sum_{k=0}^{3}\left(P S_{k}^{l}+P C^{w l_{k}}+P C^{l_{k} l_{k}}\right) \cdot\left(\Gamma_{k}+T_{l}\right) \\
& +\sum_{j=0}^{2} P C^{l_{3} l_{j}} \cdot\left(\Gamma_{3}+T_{l}\right)+\sum_{s=0}^{1} P C^{l_{2} l_{s}} \cdot\left(\Gamma_{2}+T_{l}\right) \\
& +P C^{l_{1} l_{0}} \cdot\left(\Gamma_{1}+T_{l}\right)+P C^{w w} \cdot T_{c}^{w w}+P_{\mathrm{idle}} \sigma,
\end{aligned}
$$

where, $N_{i}$ is the number of Wi-Fi frames transmitted in the TXOP of traffic Class $i$; $T_{s}^{w}$ and $T_{c}^{w w}$ are respectively the time durations of a successful and colliding Wi-Fi transmissions, given by

$$
\begin{aligned}
T_{s}^{w}= & T_{p h y}+\frac{D_{\mathrm{mac}}}{B R}+\frac{D_{\text {data }}}{D R}+2 \cdot \operatorname{SIFS}+\frac{D_{\text {ack }}}{B R} \\
T_{c}^{w w}= & \min \left[\mathrm{SIFS}+\sigma \mathrm{AIFSN}_{i}\right]+T_{p h y}+\frac{D_{\text {mac }}}{B R} \\
& +\frac{D_{\text {data }}}{D R}+\text { ACK }_{\text {Timeout }} ;
\end{aligned}
$$

where $T_{p h y}$ is the physical layer overhead; $D_{\text {mac }}, D_{\text {data }}$ and $D_{\text {ack }}$ are respectively the size of the mac header, data payload, and ack frame; $D R$ and $B R$ are the data and base transmission rates. In (15), the parameter $\Gamma_{i}$ is the maximum duration of an LAA transmission of Class $i$ (i.e., the LAA equivalent of the Wi-Fi TXOP). $T_{l}$ is the average delay between the time at which an LAA station gains access to the channel using the CSMA/CA mechanism and the beginning of an LAA time slot.

\section{Throughput of Wi-Fi and LAA in a Coexistence Scenario}

For both Wi-Fi and LAA, the throughput can be calculated as the ratio between the average duration of a successful transmission and the average duration of the contention slot $T_{\text {cs }}$. In the case of $\mathrm{Wi}-\mathrm{Fi}$, a collision causes the first packet of a TXOP to be lost. Without an acknowledgment, the transmitting station starts the backoff process before the end of its TXOP. Hence, the only event which results in the transmission of data is a successful transmission $P S_{i}^{w}$. The throughput of Class $i$ of $\mathrm{Wi}-\mathrm{Fi}$ is given by

$$
T h_{i}^{w}=\frac{P S_{i}^{w} N_{i} \frac{D_{\mathrm{data}}}{D R}}{T_{\mathrm{cs}}} .
$$

In the case of LAA, a collision causes the sub-frames which overlap in time with the Wi-Fi transmission to be lost. Each sub-frame comprises of 14 OFDM symbols of which we assume that the first one only carries control information. The throughput of LAA for Class $i$ is given by

$$
\begin{aligned}
T h_{i}^{l}= & \frac{13}{14} \frac{1}{T_{\mathrm{cs}}}\left[P S_{i}^{l} \Gamma_{i}+P C_{i}^{w l}\left(\Gamma_{i}-P_{\mathrm{fc}} \cdot T_{\text {sframe }}\right)\right. \\
& \left.+\sum_{k<i} P C^{l_{i} l_{k}}\left(\Gamma_{i}-\Gamma_{k}\right)\right],
\end{aligned}
$$

where $T_{\text {sframe }}$ is the duration of an LTE sub-frame, and $P_{\mathrm{fc}}$ is the probability that a Wi-Fi transmission causes the first sub-frame to be lost.

\section{E. Fairness Calculation}

To measure the throughput fairness between $\mathrm{Wi}-\mathrm{Fi}$ and LAA, we use Jain's Fairness Index. Jain's Fairness Index is defined as:

$$
\mathcal{J}\left(x_{1}, x_{2}, \ldots, x_{n}\right)=\frac{\left(\sum_{k=1}^{n} x_{i}\right)^{2}}{n \cdot \sum_{k=1}^{n} x_{i}^{2}}
$$




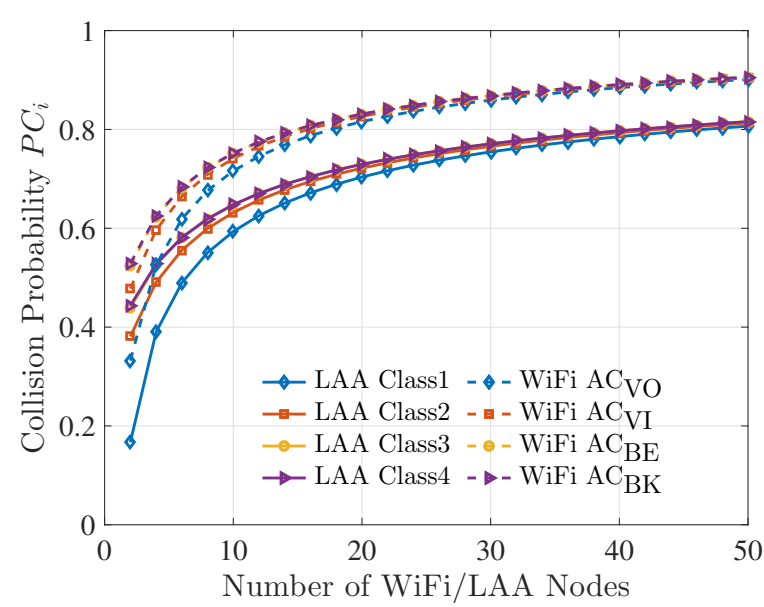

(a) Collision probability of LAA and Wi-Fi.

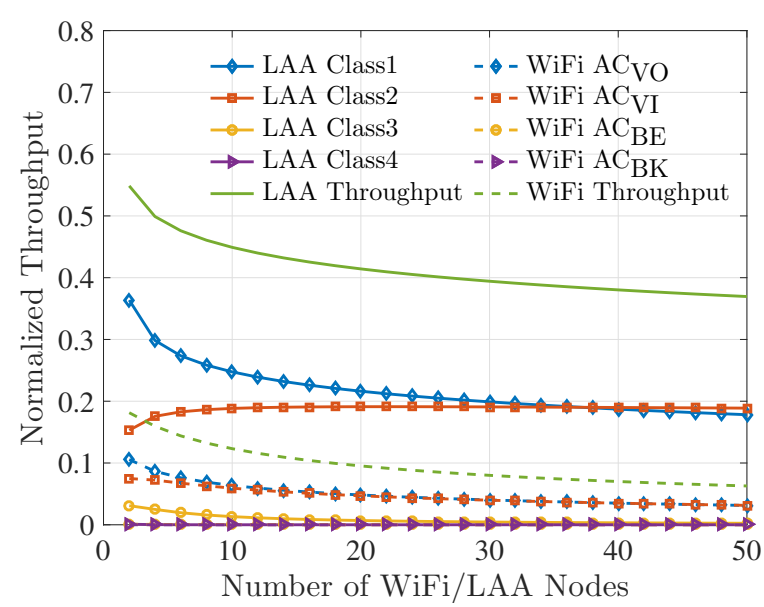

(b) Throughput for Each Priority Class.

Fig. 2: LAA and Wi-Fi coexistence performance with priority classes, $n_{w}=n_{l}=\mathrm{N} / 2$, where $\mathrm{N}$ is the total number of nodes in the network).

where $x_{i}$ is the ratio between the actual throughput and the optimal throughput. We define the fair throughput $T h_{f}$, as the normalized Wi-Fi throughput when LAA is not present. We want to measure the difference in the normalized throughput of the Wi-Fi nodes before and after half of the Wi-Fi nodes are replaced with LAA nodes of same type and same traffic. To achieve the desired fairness, the normalized throughput of Wi-Fi after the substitution should be greater or equal to the normalized throughput before the substitution. That is, the performance of Wi-Fi nodes should not be negatively impacted by the introduction of LAA nodes more than if the LAA nodes were communicating using Wi-Fi.

Let $x_{1}$ be the ratio between the normalized throughput of Wi-Fi when LAA is not present and the fair throughout $T h_{f}$; in which case $x_{1}=1$. After replacing half of the Wi-Fi nodes with equivalent LAA nodes, let $x_{2}$ be the ratio between the normalized throughput of Wi-Fi and the fair throughput $T h_{f}$. We can calculate Jain's Fairness Index as

$$
\mathcal{J}\left(x_{1}, x_{2}\right)=\frac{\left(x_{1}+x_{2}\right)^{2}}{2 \cdot\left(x_{1}^{2}+x_{2}^{2}\right)}=\frac{\left(1+x_{2}\right)^{2}}{2 \cdot\left(1+x_{2}^{2}\right)}
$$

\section{F. Extension to Wi-Fi with RTS/CTS}

With few modifications, the proposed analytical model is adapted to cover the case in which Wi-Fi uses the RTS/CTS mechanism. For this, the following aspects of the model need to be modified:

1) The maximum number of retransmissions $\left(M_{i}\right)$ for all Wi-Fi classes is increased to 7;

2) $P_{f c}$, the probability that a collision between Wi-Fi and LAA occurs before the beginning of an LAA slot is equal to the ratio of a RTS frame transmission time and an LTE slot time $(0.5 \mathrm{~ms})$.

3) The expressions for $T_{c}^{w w}$ and $T_{E}$ are given by (22) and (23);

$$
\begin{aligned}
T_{c}^{w w}= & \min \left[\mathrm{SIFS}+\sigma \operatorname{AIFSN}_{i}\right]+T_{p h y}+\frac{D_{\mathrm{rts}}}{B R} \\
& +\mathrm{RTS}_{\text {Timeout }} .
\end{aligned}
$$

TABLE IV: Wi-Fi \& LAA Parameters

\begin{tabular}{|c|c|c|c|}
\hline \multicolumn{2}{|c|}{ Wi-Fi parameters } & \multicolumn{2}{c|}{ LAA Parameters } \\
\hline \hline Parameter & Value & Parameter & Value \\
\hline$D R, B R$ & $54 \mathrm{Mbps}, 6 \mathrm{Mbps}$ & Data Rate & $70.2 \mathrm{Mbps}$ \\
\hline$\sigma, \mathrm{SIFS}$ & $9 \mu \mathrm{s}, 16 \mu \mathrm{s}$ & $\sigma$ & $9 \mu \mathrm{s}$ \\
\hline$D_{a c k}, D_{\text {mac }}$ & $112,272 \mathrm{bits}$ & $T_{f}$ & $16 \mu \mathrm{s}$ \\
\hline$T_{\text {phy }}, \mathrm{ACK}_{\text {Timeout }}$ & $20 \mu \mathrm{s}, 50 \mu \mathrm{s}$ & $T_{\text {sframe }}$ & $1 \mathrm{~ms}$ \\
\hline$D_{\text {data }}$ & 1470 bytes & $T_{l}$ & $0.25 \mathrm{~ms}$ \\
\hline
\end{tabular}

$$
\begin{aligned}
T_{E}= & \sum_{i=0}^{3} P S_{i}^{w} \cdot\left(\min \left[\operatorname{SIFS}+\sigma \operatorname{AIFSN}_{i}\right]+T_{p h y}\right. \\
& \left.+\frac{D_{r t s}}{B R}+\mathrm{SIFS}+T_{p h y}+\frac{D_{c t s}}{B R}+T_{s}^{w} \cdot N_{i}-\mathrm{SIFS}\right) \\
& +\sum_{k=0}^{3}\left(P S_{k}^{l}+P C^{w l_{k}}+P C^{l_{k} l_{k}}\right) \cdot\left(\Gamma_{k}+T_{l}\right) \\
& +\sum_{j=0}^{2} P C^{l_{3} l_{j}}\left(\Gamma_{3}+T_{l}\right)+\sum_{r=0}^{1} P C^{l_{2} l_{r}} \cdot\left(\Gamma_{2}+T_{l}\right) \\
& +P C^{l_{1} l_{0}}\left(\Gamma_{1}+T_{l}\right)+P C^{w w} \cdot T_{c}^{w w}+P_{i d l e} \sigma .
\end{aligned}
$$

\section{Performance Evaluation}

The proposed mathematical model is used to study the performance of both Wi-Fi and LAA priority classes. The Markov Chain model for EDCA on which our own proposed model is based was validated by means of simulations in [16]. The PHY and MAC layer parameters for Wi-Fi and LAA networks are reported in Table IV, while the EDCA and LAA class parameters are given in Table I and II, respectively.

Fig. 2a shows the collision probability of LAA and Wi-Fi priority classes. From the figure, we can clearly observe that the collision probability of LAA is always lower than Wi-Fi. This should not be very surprising as the collision probabilities calculated in (5) and (6) depend on the transmission probabilities $\tau_{i}$ which are a function of the backoff parameters. Hence, for similar choice of backoff parameters, the collision probability of Wi-Fi and LAA would have also been similar 


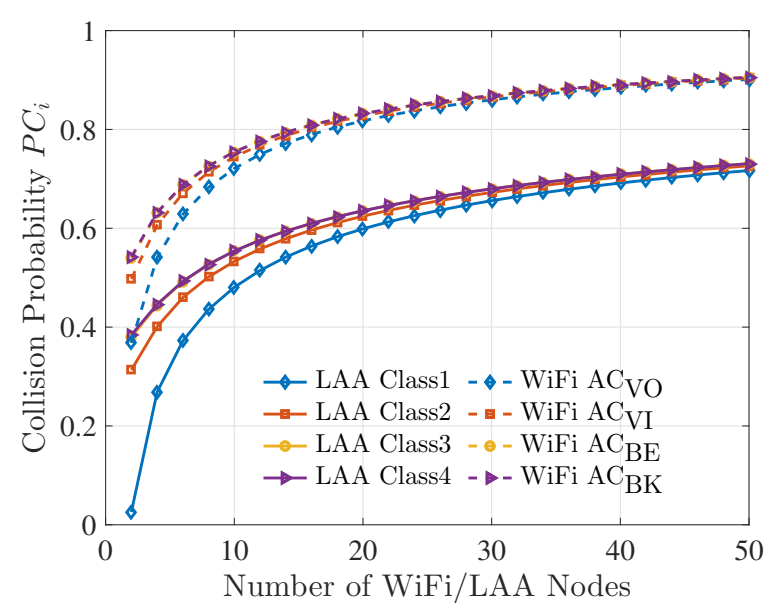

(a) Collision probability of LAA and Wi-Fi.

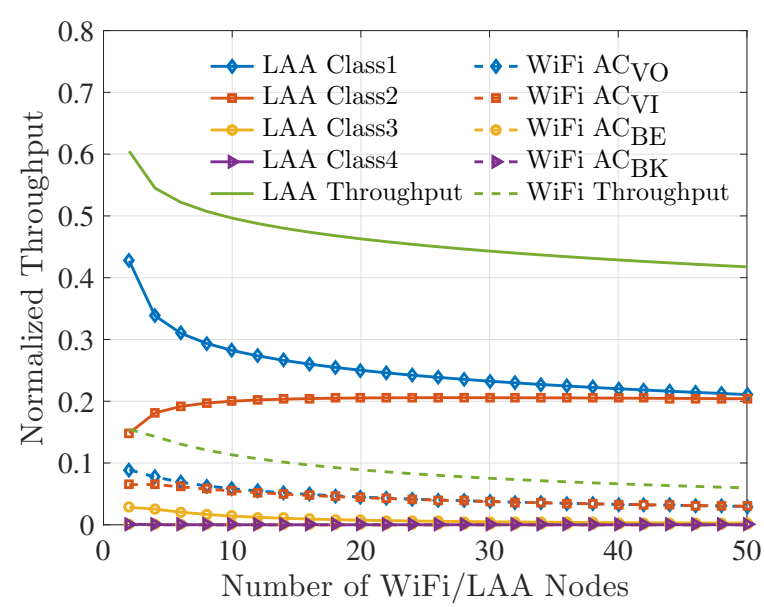

(b) Throughput for each priority class.

Fig. 3: LAA and Wi-Fi coexistence performance with RTS/CTS enabled, $n_{w}=n_{l}=\mathrm{N} / 2$, where $\mathrm{N}$ is the total number of nodes in the network).

if it was not for the fact that LAA transmissions are only affected on average by a fraction $P_{\mathrm{fc}}$ of the collisions with Wi-Fi.

Fig. $2 b$ shows the normalized throughputs for each access category in LAA and Wi-Fi coexistence scenario. The behaviour of Wi-Fi access categories is as expected i.e., higher priority classes have more throughput compared to lower priority classes. We note that for each priority class, Wi-Fi has lower throughput than LAA. The main reason for this is the way collisions are handled in Wi-Fi compared to LAA. In Wi-Fi, a collision causes the station to prematurely end the TXOP and thus refraining from transmitting any further frame; whereas LAA continues transmitting until the end of the transmission duration. This allows LAA to use the channel for longer time and successfully transmit some of its subframes even in case of a collision with Wi-Fi. Surprisingly, the throughput of LAA Class 1 is higher than Class 2 for medium to high load. Hence, the LAA access categories are not performing as expected i.e., there is a priority inversion between Class 1 and Class 2 at high load. This behaviour can be explained by considering that for medium to high load the transmission probability of Class 1 is much higher than Class 2. Hence, there is a high probability of collision between two or more Class 1 transmissions, causing the complete loss of the data transmitted by Class 1 , whereas a single Class 2 colliding with any number of Class 1 transmissions would still be able to successfully transmit $33 \%$ of its data. Finally, to check the fairness of LAA with Wi-Fi, we first considered only Wi-Fi nodes in the network (i.e. WW scenario) and then replaced $50 \%$ of Wi-Fi nodes with LAA nodes (i.e. WL scenario). From Fig. 4a, it is clear that the performance of Wi-Fi degrades when LAA nodes are introduced. The main reason for such behaviour is that the transmission duration of LAA is much longer than Wi-Fi. Furthermore, the collision of LAA with Wi-Fi causes the loss of all Wi-Fi data, whereas a portion of LAA data still successfully received as only the first sub-frame is lost.

\section{A. Coexistence with RTS/CTS Enabled in Wi-Fi}

The RTS/CTS can be optionally employed by Wi-Fi nodes to combat the effect of the hidden nodes problem. Given that in our system model, we assume that there are no hidden nodes, the benefit of using RTS/CTS is limited to a decrease in the duration of collisions. We modified the model as described in Section III-F to study the effects of the RTS/CTS on the fairness between Wi-Fi and LAA. Because of the small size of a RTS frame, the probability that a collision between a RTS frame and an LAA transmission occurs before the beginning of an LAA sub-frame is higher than if a larger data packet was transmitted. This leads to a decrease in the collision probability of LAA, clearly observed by comparing Fig. 3a to Fig. 2a. Comparing Fig. 4b to Fig. 4a, we see that when only Wi-Fi is present (i.e., WW scenario), the RTS/CTS increases the throughput of Wi-Fi for large number of nodes. In the coexistence scenario (i.e., LW scenario), using RTS/CTS does not increase the throughput of Wi-Fi nodes. If RTS/CTS is used with a number of Wi-Fi and LAA nodes above 20, the total throughput in scenario LW is lower than in WW.

\section{B. Impact of LAA Transmission Duration on Fairness}

Wi-Fi is mostly affected by a collision with LAA as it interrupts the transmission and loses the channel access opportunity, whereas LAA continues transmitting till the end of its transmission opportunity. This difference in behaviour results in a higher throughput of LAA compared to Wi-Fi and in poor performance of Wi-Fi in LAA - Wi-Fi scenario compared to Wi-Fi only scenario. Thus, to improve the fairness, we propose to reduce the LAA transmission duration for Class 1 and Class 2 to one millisecond. The Jain's fairness index with the regular and the proposed transmission duration are shown in Fig. 5. The throughput fairness of Wi-Fi is one for the case of only Wi-Fi nodes (i.e., reference case) and it is reduced to an average of 0.84 for the case of Wi-Fi and LAA nodes. With 


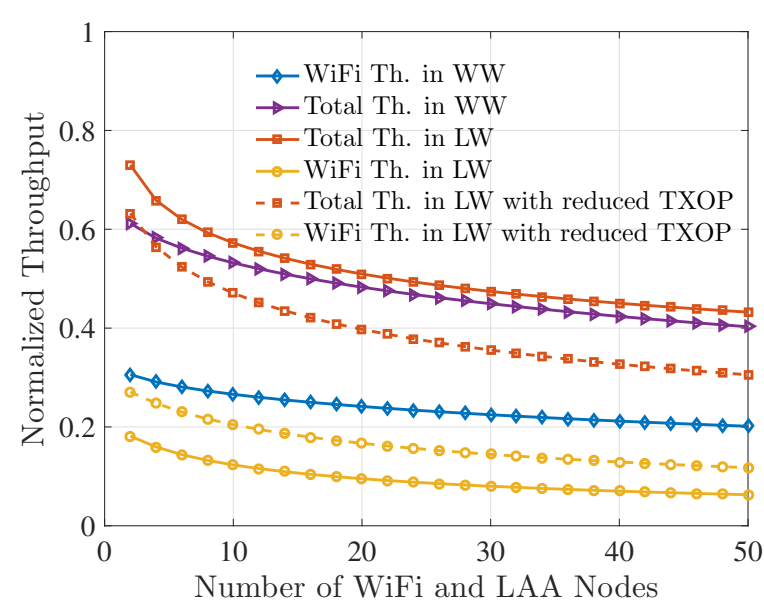

(a) Throughput without RTS/CTS.

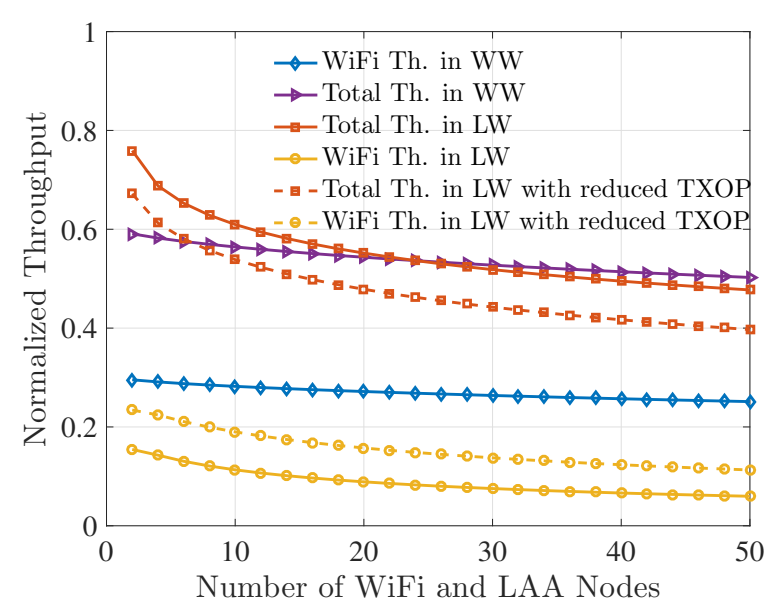

(b) Throughput with RTS/CTS.

Fig. 4: Wi-Fi and Total Throughput (i.e., Wi-Fi + LAA) in Wi-Fi only scenario (WW) and coexistence scenario (LW).

the proposed approach (i.e., with reduced LAA transmission duration) the fairness is increased to an average of 0.96 for the case of Wi-Fi and LAA nodes. This increase in fairness comes at the cost of reduction in total throughput between 13 and 30\%, as shown in Fig. 4a. Similar increase in the fairness index is visible even when the RTS/CTS mechanism is used as shown in Fig. $4 \mathrm{~b}$.

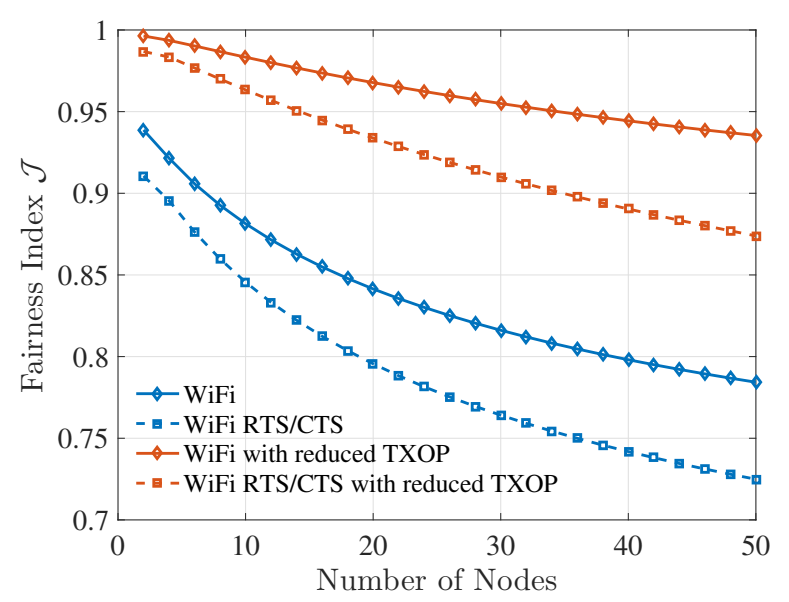

Fig. 5: Wi-Fi and LAA Fairness.

\section{CONClusions}

In this work, a mathematical model is proposed to study the coexistence between Wi-Fi and LAA users. The model allows to calculate the throughput of each of the four priority classes defined by LAA and EDCA. In calculating the throughput, particular attention was given to correctly account for partial collision of LAA transmissions and Wi-Fi. The results show that although the backoff parameters of Wi-Fi and LAA are similar, the latter is clearly unfair to the first. We found that reason behind this is the different channel access durations of Wi-Fi and LAA transmissions. And on the fact that channel access attempts resulting in collisions can still allow LAA to successfully deliver part of the transmitted subframes, whereas Wi-Fi transmission would fail. In the future, we plan to analyse the QoS performance by including the average delay of each Wi-Fi and LAA priority class in the model.

\section{REFERENCES}

[1] "LTE in Unlicensed Spectrum : Harmonious Coexistence with Wi-Fi," Qualcomm white paper, June 2014.

[2] 3GPP, "TSGRAN; Study on Licensed-Assisted Access to Unlicensed Spectrum," Tech. Rep. TR 36.889 V13.0.0, June 2015.

[3] C. Cano and D. J. Leith, "Unlicensed LTE/WiFi Coexistence: Is LBT Inherently Fairer Than CSAT?" in IEEE ICC, July 2016.

[4] "LTE-U SDL Coexistence Specifications," LTE-U Forum [Online]. Available: http://www.lteuforum.org/documents.html, 2015.

[5] "IEEE Standard for Information technology-Telecommunications and information exchange between systems Local and metropolitan area networks-Specific requirements Part 11: Wireless LAN Medium Access Control (MAC) and Physical Layer (PHY) Specifications," March 2012, pp. 1-2793.

[6] A. V. Kini, L. Canonne-Velasquez, M. Hosseinian, M. Rudolf, and J. Stern-Berkowitz, "Wi-Fi-LAA coexistence: Design and evaluation of listen before talk for LAA," in IEEE CISS 2016, pp. 157-162.

[7] S. Dama, A. Kumar, and K. Kuchi, "Performance evaluation of laalbt based lte and wlan's co-existence in unlicensed spectrum," in IEEE Globecom Workshops, 2015, pp. 1-6.

[8] C. Casetti, "Coexistence of IEEE $802.11 \mathrm{n}$ and Licensed-Assisted Access devices using Listen-before-Talk techniques," in IEEE CCNC, 2016, pp. 562-567.

[9] C. Chen, R. Ratasuk, and A. Ghosh, "Downlink performance analysis of LTE and WiFi coexistence in unlicensed bands with a simple listenbefore-talk scheme," in IEEE VTC Spring, 2015, pp. 1-5.

[10] G. J. Sutton, R. P. Liu, and Y. J. Guo, "Delay and Reliability of LoadBased Listen-Before-Talk in LAA," vol. 6. IEEE, 2018, pp. 6171-6182.

[11] Y. Gao, X. Chu, and J. Zhang, "Performance analysis of LAA and WiFi coexistence in unlicensed spectrum based on Markov chain," in IEEE Globecom, 2016, pp. 1-6.

[12] "Broadband Radio Access Networks (BRAN); 5 GHz high performance RLAN; Harmonized EN covering the essential requirements of article 3.2 of the R\&TTE Directive," in ETSI EN 301893 V1.8.1, 2015.

[13] R. Zhang, M. Wang, L. X. Cai, Z. Zheng, X. Shen, and L.-L. Xie, "LTEunlicensed: the future of spectrum aggregation for cellular networks," vol. 22, no. 3. IEEE, 2015, pp. 150-159.

[14] G. Bianchi, "Performance analysis of the IEEE 802.11 distributed coordination function," in IEEE Journal on Selected Areas in Commun., vol. 18, no. 3. IEEE, 2000, pp. 535-547.

[15] M. Mehrnoush and S. Roy, "On the Fairness of Wi-Fi and LTE-LAA Coexistence," arXiv preprint arXiv:1805.03501, 2018.

[16] K. Kosek-Szott, M. Natkaniec, and A. R. Pach, "A simple but accurate throughput model for IEEE 802.11 EDCA in saturation and nonsaturation conditions," in Computer Networks, vol. 55, no. 3. Elsevier, 2011, pp. 622-635. 